\title{
Verrucous Spitz Nevus in a Japanese Female
}

\author{
Rie Honda $^{a}$ Yuka Iino $^{a}$ Shusaku Ito $^{a}$ Masaru Tanaka $^{b}$ \\ ${ }^{a}$ Division of Dermatology, Hitachi General Hospital, Hitachi, and ${ }^{b}$ Department of \\ Dermatology, Tokyo Woman's Medical University Medical Center East, Tokyo, Japan
}

\section{Key Words}

Spitz nevus · Dermoscopy $\cdot$ Melanoma $\cdot$ Verruca vulgaris $\cdot$ Dermatofibroma

\begin{abstract}
A 23-year-old female presented with a reddish-brown dome-shaped hyperkeratotic nodule of $11 \times 10 \mathrm{~mm}$ on the left lower leg. Dermoscopic examination demonstrated a prominent whitish scaly area with ring-like appearance, pinkish-white structureless areas, a few milia-like cysts, dotted and glomerular vessels, and light brown globules. The lesion was completely excised under the diagnosis of verruca vulgaris or dermatofibroma. Histopathological examination revealed a well-circumscribed symmetric lesion with hyperkeratosis, acanthosis, and pseudohorn cysts. The lesion was composed of spindle-shaped and epithelioid melanocytes with large cytoplasm arranged in confluent nests surrounded by stromal fibrosis. Mitotic figures and Kamino bodies were absent. Moderate proliferation of capillaries was found in the papillary dermis. Immunohistochemical staining with melan-A or S-100 was positive for tumor cells, but the staining with HMB-45 was negative. Melan-A staining was weaker in the deeply situated cells than in the superficial ones, which is known as stratification. Finally, we made a diagnosis of verrucous Spitz nevus based on these findings. We should have been aware of the entity of verrucous Spitz nevus as a variant of Spitz nevus and its dermoscopic features in order to reach a correct diagnosis before excision.
\end{abstract}

(C) 2013 S. Karger AG, Basel

\section{Case Presentation}

A 23-year-old Japanese female presented with a pigmented skin lesion on the left lower leg. It had been noted for 5 months. She had no specific symptoms. Physical examination revealed a reddish-brown dome-shaped hyperkeratotic nodule of $11 \times 10 \mathrm{~mm}$ with a sharply 
Honda et al.: Verrucous Spitz Nevus in a Japanese Female

demarcated, symmetrical border (fig. 1a). Dermoscopic examination demonstrated prominent whitish scaly areas of ring-like appearance, pinkish-white structureless areas, a few milia-like cysts, dotted and glomerular vessels, light brown globules, and dotted hemorrhages (fig. 1b). The lesion was completely excised under the diagnosis of verruca vulgaris or dermatofibroma.

Histopathologic study revealed a well-circumscribed symmetric lesion with no lateral extension of individual melanocytic proliferation. Hyperkeratosis, acanthosis, and pseudohorn cysts were observed (fig. 2a). There were confluent nests with peripheral clefts composed of spindle-shaped and epithelioid melanocytes with large cytoplasm at the dermal-epidermal junction and in the papillary dermis (fig. 2b). There was stromal fibrosis surrounding the nests (fig. 2c). Mitotic figures and Kamino bodies were absent. Moderate proliferation of capillaries in the papillary dermis was observed (fig. 2d). There were perivascular cell infiltration and maturation of nevus cells at the bottom of the lesion. Immunohistochemical staining with melan-A or S-100 was positive for tumor cells, but the staining with HMB-45 was negative. Melan-A was weaker in the deeply situated cells than in the superficial ones (fig. 3a), and nests ascending up to the stratum corneum appeared as transepidermal elimination (fig. $3 \mathrm{~b}$ ). We eventually made a diagnosis of verrucous Spitz nevus based on these findings.

\section{Discussion}

Spitz nevus is usually solitary and is encountered most commonly in the lower extremities and face. In most instances, it consists of a dome-shaped, hairless, small pink nodule. The size is usually less than $1 \mathrm{~cm}$ in diameter. However, because of few specific clinical features, it is often difficult to make a correct diagnosis of Spitz nevus. Accordingly, Spitz nevus is sometimes clinically mistaken for other skin tumors such as hemangioma, verruca vulgaris, dermatofibroma, and so on. It looks pinkish in color because of a scarcity of melanin, and some lesions are associated with vascularization of the stroma [1]. The major histological diagnostic criteria include the cell type (epithelioid and/or spindle cells), the symmetrical appearance of the lesion, maturation of nevus cells at the bottom of the lesion, the lack of pagetoid spread of single melanocytes, and the presence of coalescent eosinophilic globules (Kamino bodies). Minor diagnostic criteria include the presence of junctional cleavage, pseudoepitheliomatous hyperplasia, an absence of epidermal consumption, superficial dermal edema and telangiectasia, giant nevus cells, and an absence of nuclear pleomorphism. Other histological changes that might rarely occur are stromal fibrosis, hyalinization of the stroma, and stromal mucin deposition [2].

The histopathologic differential diagnosis of Spitzoid tumors represents a difficult challenge. In our patient, the findings of acanthosis and hyperkeratosis favored the diagnosis of Spitz nevus rather than melanoma. In addition, the proliferation pattern in the dermis was not sheet-like, but rather mainly confluent nests with stromal fibrosis, which also supported a diagnosis of Spitz nevus. Because of continuous sheet-like proliferation, stromal fibrosis is absent in melanoma. By immunohistochemistry, melan-A showed weaker staining in the deeply situated cells than in the superficial ones. This pattern, known as stratification, would not be seen in melanomas [2]. On the basis of these clinicopathological features, a final diagnosis of verrucous Spitz nevus was established.

On dermoscopy, pink Spitz nevus typically exhibits little pigmentation and features a characteristic dotted vascular pattern [3]. Indeed, a dotted vessel pattern has been observed in $77.8 \%$ of Spitz nevus cases [4]. A negative pigment network surrounding dotted vessels 
Honda et al.: Verrucous Spitz Nevus in a Japanese Female

has also been seen in $28.8 \%$ of cases [5]. Pigmented Spitz nevus (Reed nevus) shows very different appearances on dermoscopy; it typically exhibits either a 'starburst' or a 'globular' pattern [3].

The present case showed the characteristic dermoscopic pattern of verruca vulgaris, such as ring-like whitish scaly areas and dotted vessels. In addition, the presence of a central white patch was considered as the most common dermoscopic pattern in dermatofibroma. In fact, the dermoscopic similarity between Spitz nevus and dermatofibroma is sometimes a cause of misdiagnosis [6]. With the dermoscopic patterns in our case, we should have borne in mind some other differential diagnoses such as seborrheic keratosis, eccrine poroma, and Spitz nevus, along with verruca vulgaris and dermatofibroma. The presence of milia-like cysts might have suggested a diagnosis of seborrheic keratosis. Moreover, pinkish-white structureless areas with dotted hairpin or glomerular vessels are typical of the dermoscopic pattern in eccrine poroma. However, dotted vessels correspond to the proliferation of capillaries in the papillary dermis, which played a key role in the correct diagnosis of Spitz nevus. Moreover, dotted hemorrhages might often be seen in verruca vulgaris due to prominent papillomatosis, which is, on the contrary, not usually seen in Spitz nevus. The histological difference between Spitz nevus and verruca vulgaris would correspond to the different pattern on dermoscopy and, therefore, it might be a key feature for the differentiation of Spitz nevus from verruca vulgaris. If we had initially interpreted the dermoscopic findings more carefully, we might have included a correct diagnosis of verrucous Spitz nevus, at least in the list of differential diagnoses.

\section{Disclosure Statement}

The authors have no conflicts of interest to disclose.

\section{References}

1 Elder D, Elenitsas R, Murphy G, et al: Benign pigmented lesions and malignant melanoma; in Elder DE, Johnson B Jr, Elenitsas R (eds): Lever's Histopathology of the Skin, ed 9. Philadelphia, Lippincott, 2005, pp 735-740.

2 Weedon D: Lentigines, nevi, and melanomas; in Weedon D: Weedon's Skin Pathology, ed 3. London, Churchill Livingstone, 2010, pp 721-725.

-3 Luo S, Sepehr A, Tsao H: Spitz nevi and other Spitzoid lesions part I. Background and diagnoses. J Am Acad Dermatol 2011;65:1073-1084.

4 Argenziano G, Zalaudek I, Corona R, et al: Vascular structures in skin tumors: a dermoscopy study. Arch Dermatol 2004;140:1485-1489.

5 Pizzichetta MA, Talamini R, Marghoob AA, et al: Negative pigment network: an additional dermoscopic feature for the diagnosis of melanoma. J Am Acad Dermatol 2013;68:552-559.

-6 Moreno-Ramirez D, Rios-Martin JJ, Ferrandiz L, et al: Dermoscopic appearance of dermatofibroma-like Spitz nevus. J Am Acad Dermatol 2009;61:910-912. 


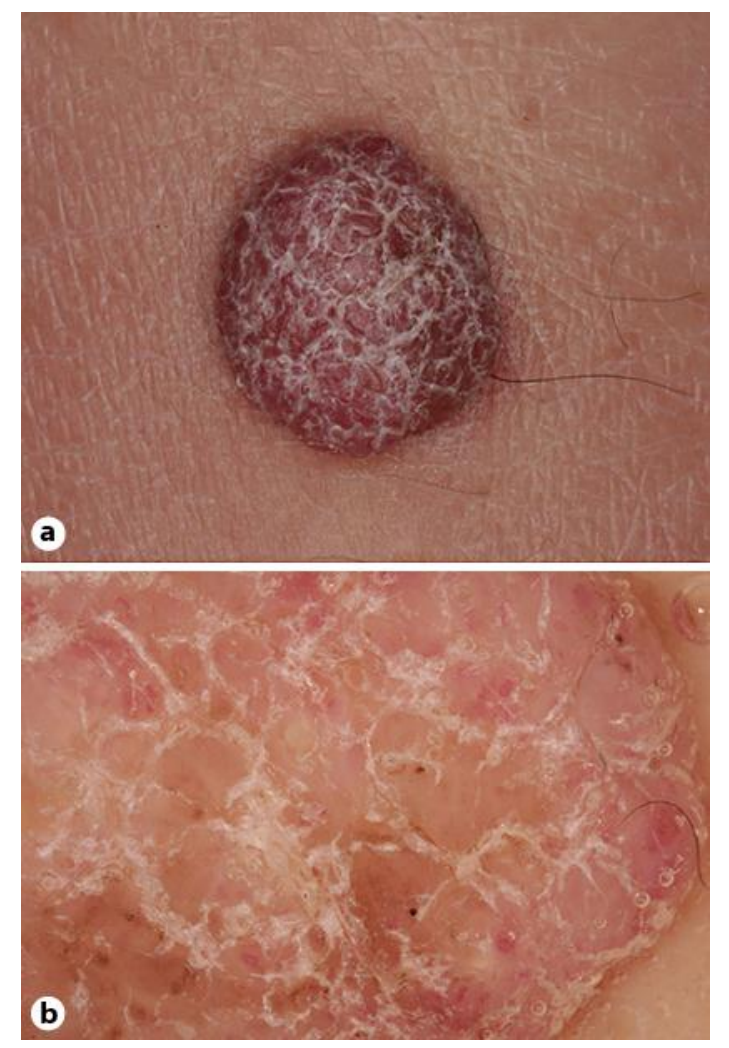

Fig. 1. a A reddish-brown dome-shaped hyperkeratotic nodule on the left lower leg. $\mathbf{b}$ The lesion showed a whitish scaly area, pinkish-white structureless areas, a few milia-like cysts, dotted and glomerular vessels, light brown globules, and dotted hemorrhages on dermoscopy. 


\section{Case Reports in Dermatology}

\begin{tabular}{l|l}
\hline Case Rep Dermatol 2013;5:304-308 \\
\hline DOI: $10.1159 / 000356470$ & $\begin{array}{l}\text { C 2013 S. Karger AG, Basel } \\
\text { www.karger.com/cde }\end{array}$ \\
\hline
\end{tabular}

Honda et al.: Verrucous Spitz Nevus in a Japanese Female
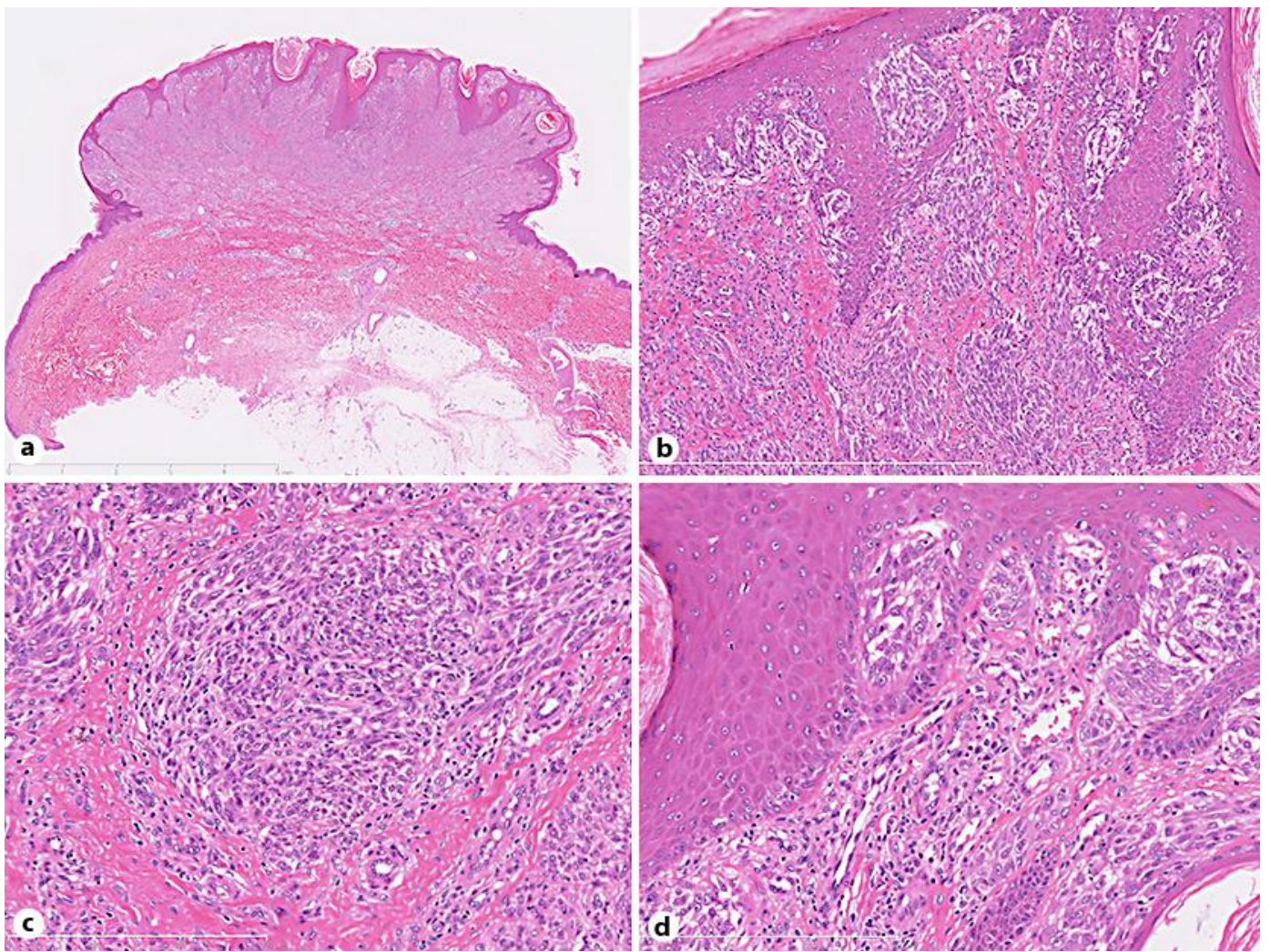

Fig. 2. a Panoramic view revealed a well-circumscribed symmetric lesion with hyperkeratosis, acanthosis, and pseudohorn cysts. $\mathbf{b}$ The lesion was composed of spindle-shaped and epithelioid melanocytes with large cytoplasm arranged in confluent nests with peripheral clefts. c There was stromal fibrosis surrounding the nests. $\mathbf{d}$ At the papillary layer, proliferation of capillary could be found. HE.

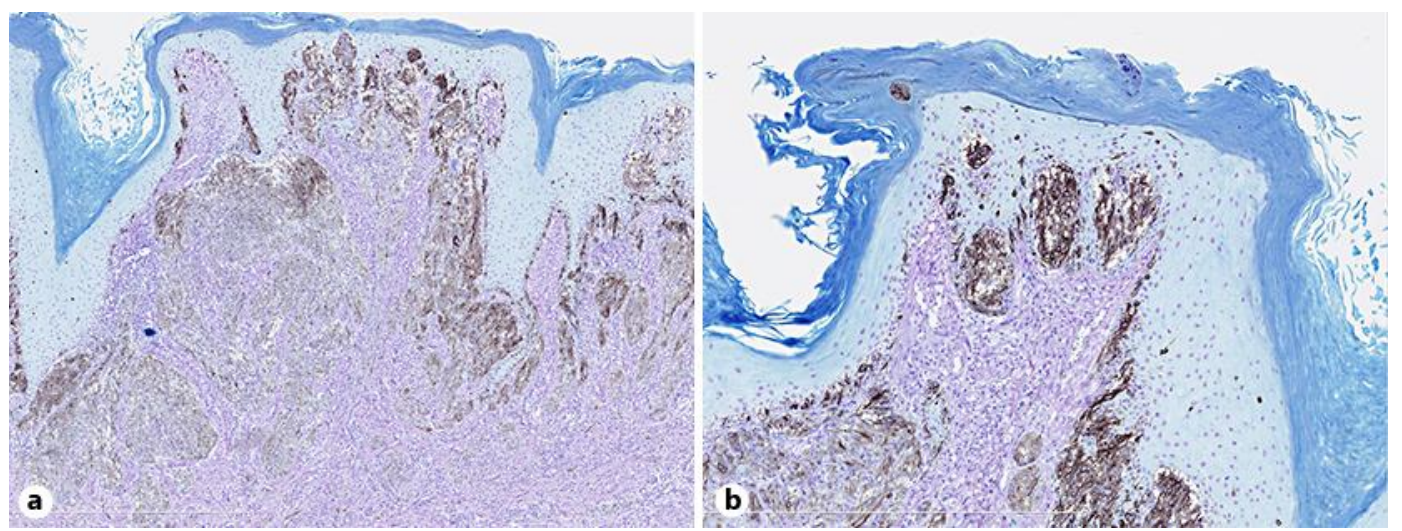

Fig. 3. a Melan-A staining showed weaker findings in the deeply situated cells than in the superficial cells. b Nests were partly ascending into the stratum corneum. Melan-A staining. 